\title{
Yasargil higway in neurosurgery (for the nineteen years old of my teacher Mahmut Gazi YASARGIL)
}

\begin{abstract}
My Teacher Professor Yasargil is very famous doctor, mentor, surgeon, lider, scientist, researcher, inventor and teacher of last Century, not only in Neurosurgery, but also in Neuroscience of the World. His very important Neurosurgical Way and thecniques, the pterional trans Sylvian approach, I called as "Yasargil Higway" is commonly employed in surgery of the anterior circulation and upper basilar artery aneurysms, as well as for the tumors of orbital, retroorbital, sellar, chiasmatic, sub frontal and prepontine areas and lesions around the sella especially for lesions behind the clivus. Also tumors arising from the medial sphenoid ridge, the superior orbital fissure, the anteromedial temporal surface, or the cavernous sinus region are approached through a pterional exposure.

The modification of surgical technique is based on the experience, training and observation of the neurosurgeon. One technique is not necessarily better than another. Regardless of the surgical technique, the end results depend on a rigorous, methodical, systematic, and step by-step approach to the target, securing it with minimal injury to surrounding structures.

In this article, according to the personal experiences of Ismail Hakk1 Aydin, MD (the Author), it was analyzed the Intraoperative anatomical findings of the Sylvian vein and fissure, lenticulostriatal artery, olfactory nerve, and recurrent artery of Heubnerand showed the surgical pitfalls and steps in personal cases with different diagnoses that were operated on with the pterional approach in Yasargil Higway. All patients were operated on by the Author. The findings were recorded during surgical interventions and through the slides and videotapes of the operations. Also, we have stressed the preservation of the front temporal branch of the facial nerve, the delicate retraction of frontal lobe, the cottonoid retraction in temporal lobe and the preservation of olfactory nerve functions.
\end{abstract}

Keywords: yasargil surgical highway, frontal branch of facial nerve, sylvian dissection, brain retraction, olfactory nerve, dural artery, heubner sartery
Volume I Issue 2 - 2014

Ismail Hakki Aydin

Department of Neurosurgery, Istanbul Aydin University, Turkey

Correspondence: Ismail Hakki Aydin, Cumhuriyet Cadd 92, Tepekent, Buyukcekmece, 34000, Istanbul, Turkey, Tel +90-532232-8I-24,Email bircis@gmail.com

Received: June 23, 2014 | Published: June 25, 2014

\section{Introduction}

According to our article data that was published in 1995, ${ }^{1}$ all steps of Pterional Yasargil Higway approach reevaluated and gold advices reviewed step by step in this paper. Different surgical methods have so far been reported for accesses to parasellar and sellar areas. ${ }^{1-10}$ The sub temporal approach was first described by Drake in $1961 .{ }^{11}$ Yasargil introduced the frontotemporosphenoidal craniotomy, which was named as a pterional approach in 1969. I got this Yasargil's approach named as "Yasargil Surgical Highway". Today, most neurosurgeons use one of these two basic surgical approaches, although with some minor modifications and refinements. ${ }^{1-11}$ The pterional approach is commonly employed in surgery of the anterior circulation and upper basilar artery aneurysms, as well as for the tumors of orbital. Retro orbital, sellar, chiasmatic, sub frontal, and prepontine areas and lesions around the sell a. especially for lesions behind the clivus. Also tumors arising from the medial sphenoid ridge, the superior orbital fissure. The anteromedial temporal surface. Or the cavernous sinus regions are approached through a pterional exposure. ${ }^{1}$

The surgical technique is based on the experience, training and observation of the neurosurgeon. One technique is not necessarily better than another. Regardless of the surgical techniques the end results will depend on a rigorous, methodical, systematic, and stepby-step approach to the target, securing it with minimal injury to surrounding structures. Surgical steps in Yasargil Higway are the patient positioning, the preservation of the front temporal branch of the facial nerve, craniotomy, Sylvian dissection and variations, retraction of frontal and temporal lobes, preservation of the lenticulostriatal arteries, preservation of the dural artery, preservation of the olfactory nerve and preservation of the Heubnerartery.

Steps:

i. The patient positioning.

ii. The preservation of the front temporal branch of the facial nerve.

iii. Craniotomy.

iv. Sylvian dissection and variations.

v. Delicate dissection of Sylvian vein and cistern.

vi. Retraction of frontal and temporal lobes.

vii. Preservation of the lenticulostriatal arteries.

viii. Importance of Dural artery.

ix. Preservation of the olfactory nerve.

x. Preservation of the Heubner artery.

Different surgical methods have so far been reported on the accesses to parasellar and sellar areas. The sub temporal approach was first described by Drake in 1961. Yasargil introduced the 
frontotemporosphenoidal craniotomy, which was named as pterional approach in 1969. Today, most neurosurgeons use one of these two basic surgical approaches, although with some minor modifications and refinements. The pterional approach is commonly employed in the surgery of the anterior circulation and upper basilar artery aneurysms, as well as for the tumours of orbital, retroorbital, sellar, chiasmatic, sub frontal and prepontine areas and lesions around the sella especially for lesions behind the clivus. Also tumours arising from the medial sphenoid ridge, the superior orbital fissure, the anteromedial temporal surface, or the cavernous sinus region are approached through a pterional exposure. The surgical technique is based on the experience, training and observation of the neurosurgeon. One's technique is not necessarily better than another's. Regardless of the surgical technique, the end results will depend on a rigorous, methodical, systematic, and step-by-step approach to the target, securing it with minimal injury to surrounding structures..$^{1-6}$

\section{The patient positioning}

At the patient positioning, especially in the cases with ACoA aneurysm and anterior skull base lesions, proper alignment of the head is very critical and important. It allows the frontal lobe to fall away from the orbital roof after opening the Sylvian fissure, following which a direct path to the lesions of the parasellar area can be developed. Also, this positioning to minimize retraction and using CSF drainage intraoperatively are practical and cost-effective ways to increase surgical exposure.

\section{Frontotemporal branch of facial nerve}

The front temporal branch of the facial nerve is the branch that can be frequently injured in the soft tissue plane of all the approaches that involve retraction of the anterior portion of the temporalis muscle to expose the lateral cranial base of the anterior cranial fossa and of the middle fossa, especially when separate skin and temporalis muscle flap were developped during the pterional craniotomy. The methods of incising and retracting of the temporalis muscle are very important Surgical damage of this nerve, (The temporal branch of facial nerve) results in a cosmetically paralysis of the frontalis, orbicularis oculi, and corrugators supercilii muscles. The preservation of this nerve depends on a clear understanding of its relationships with the complex soft tissue anatomy of the temporal-zygomatic arch region. The temporal branch of the facial nerve pierces the parotidomasseteric fascia always below the zygomatic arch and that divides into three terminal rami. These are anterior, middle and posterior rami. The anterior ramus innervates the corrugators supercilii and orbicularis oculi muscles, and the middle one that was named as frontal ramus, innervates the frontalis muscle. The posterior ramus, for the variously developed anterior and superior auricular and tragus muscles, does not have any practical importance in man. The temporal branch of the facial nerve can show a significant variability in its coursing.

The superficial temporalis facia has two layers. The first one is the superior layer (The superficial layer) that contains fat, a large temporal vein and the temporalis branch of the facial nerve. The second is the inferior layer (The deeper layer) that covers the temporalis muscle and contains a deep temporal artery and vein. These two layers should be separately dissected to avoid injury to the frontal branch of the facial nerve. Also there is a regular large vein within the sickle shaped fat layer. The surgical separation of these two layers of the superficial temporalis facia can be identified by a small quantity of fat concealed between them. ${ }^{1,2,10}$ The damage may occur with electro coagulation of nearby vessels. The nerve may be stretched from sutures or fish- hook retractions. Also, this branch may be injured as one removes bone inferiorly from the most anterior burr hole. But, this inferior burr-hole has importance for a good exposure. The hole, near the frontozygomatic suture, should be as low as possible on the orbit. Yasargil facetiously and ironically said:'If you don't see intraorbital fat, you aren't low enough". Incomplet injury of the temporal branch of the facial nerve usually returns to function in about 6 months. In principle, it would be ideal if the brain could be left completely undisturbed while the surgical operation was carried out. ${ }^{1}$

\section{Sylvian dissection}

The arachnoid of the Sylvial cistern should be opened on the frontal side of the Sylvian vein so that they will not cross the Sylvian fissure when the frontal lobe is retracted. It must be point out that, the exact location of the Sylvian fissure medially is not always apparent and that the fissure does not always "open" in a clean arachnoidal plane, as is frequently illustrated. ${ }^{1-11}$ It is possible to see the variations of the width of the Sylvian cistern and the middle cerebral artery position in some cases. ${ }^{1}$ The herniation of the lateral fronto-orbital gyrus into the temporal lobe and the herniation of the superior temporal gyrus into the lateral fronto-orbital gyrus can be seen in some cases..$^{1-3}$ All of them are very important changes to be considered for the micro dissection plan. ${ }^{1-6}$ Coagulation of the dural fold at the superior orbital fissure is avoided for the fear of producing a sixth nerve palsy. Maximum surface exposure and minimum brain retraction are the keys to successful surgery via the pterional approach. Our goal should be to obtain the maximum possible exposure with minimal brain retraction. Yasargil has emphasized the value of opening the medical aspect of the Sylvian fissure in reducing the injury of brain retraction. Arachnoidal dissection is the real key to successful operation, especially in the cases with skull base lesions. Every neurosurgeons must know and be familiar the detailed anatomy of the subarachnoid cisterns. ${ }^{1-11}$

\section{Retraction of frontal and temporal lobes}

After the dural opening, the frontal lobe is gently and carefully retracted allowing easy access and entrance into the carotid, chiasmatic, and lamina terminali cisterns, thereby releasing CSF and providing the necessary room for easy dural tacking. The release of CSF from Sylvian, carotid, chiasmatic and interpeduncular cisterns provides a quick effective reduction of cerebral volume and facilitates the approaches to aneurysms, pituitary gland adenomas and parasellar tumours. ${ }^{1-11}$ Sometimes, in the patients with sellar or parasellar tumours, these cisterns were occluded by the mass effect and this CSF releasing manoeuver does not provide sufficient fluid discharge, necessitating opening the contra lateral cisterns to gain adequate brain decompression. ${ }^{1}$ According to Yasargil, The Subarachnoid cisterns are the roadmaps for micro neurosurgeon. Sylvian Dissection should be done with the; Inside-outside, Outside-inside, Proximal, Medial and Distal techniques, according to the Sylvian anatomical variations ${ }^{1}$ the wider the arachnoidal dissection, the better the exposure of deep structures.

The main goal of microneuro surgical operations is the ability of the surgeon to perform all the necessary manipulations through a small "Key-Hole" approach. For the mastery of tactics and techniques of this "key-hole" surgery it is absolutely necessary to be familiar with a new perspective of the anatomy of the cisternal and neurovascular system. The subarachnoid cisterns with micro technique enable the surgeons to dissect approach and expose the vascular and temporal lesions in a anatomical and natural pathway. ${ }^{1-11}$ If the arachnoid is sufficiently fine, simply a spreading action with fine bipolar forceps 
is adequate. After dural opening, the arachnoidal dissection must be accomplished by the gentle spreading of the tips bipolar forceps. Arachnoidal thickened bands and adhesions should be divided sharply and should be avoided from the traction. Blunt dissection and traction can cause in the avulsion of connective tissue and small vessels. Also, keeping of the tips of bipolar coagulation forceps, absolutely clean and moist, is very important. This prevents sticking and charring of tissue during coagulation. ${ }^{1}$ If possible, it should be avoided from the temporal lobe retraction. Otherwise, the temporal lobe retraction should be done very carefully, to not avulse and damage any of the small branches of the distal carotid, the proximal middle cerebral, and the anterior choroid arteries. Also, in the patients with the temporal lobe retraction have more epileptic seizure incidence postoperatively, than the others.

So that, more brain retraction is required during the operation in order to visualize the pathway to the lesions. Such excess brain retraction pressure can lead to postoperative brain edema and necrosis. A small bridging vein from the superficial middle cerebral vein to the sphenoparietal or cavernous sinus may need to be divided to allow retraction. In some cases the vein can be dissected from the pia to obtain adequate length to avoid injury to it. Temporal tip vein; can be a source of bleeding, because of avulsion, during temporal retraction. If possible; dissect and free it. The brain retraction must be done with minimal force. The use of multiple, narrow blades may be less injurious than a single, wide blade. Retractor blades must be released intermittently during prolonged operations to allow brain perfusion. Bipolar coagulation is very important and protects the neighbouring structures from the effect of heating. Accurate coagulation of small vessels is essential. Bleeding small vessels should be coagulated over a few millimetres length and divided. Otherwise, they retract and begin to bleed again. ${ }^{1-10}$

\section{Lenticulostriatal arteries}

Lenticulostriatal arteries in my cases were originating,

a. From one single large artery $52 \%$

b. From two large parallel arteries $35 \%$

c. As multiple branches $13 \%$

\section{Importance of dural artery}

Dural artery also has an importance that is originating, mostly from ICA, and rarely from first segment of ACA. Retraction may blanch dural artery, so it appears only to be a strand of arachnoid, and may be accidentally avulsed from the parent artery. After a local sympathectomy and the application of papaverine to the ICA, Dural artery may be a source of unrecognized bleeding.

\section{Olfactory nerve function}

The pterional approach is commonly employed in the surgery of the anterior circulation and upper basilar artery aneurysms, as well as for the tumours of orbital, retroorbital, sellar, chiasmatic, sub frontal and prepontine areas and lesions around the sella especially for those behind the clivus. Anosmia is not rare after surgery for ACoA aneurysms via either the front temporal or the interhemispheric approach. It was reported that the olfactory nerve might be pulled out of the cribriform plate as a result of excessive retraction beyond a certain limit by a retractor which is placed on the frontal lobe during the front temporal approach. It was also stated that even mild retractory pressure on cranial nerves might lead to temporary or permanent lesions. We have failed to find any clear description of the risk of damage to the olfactory nerve associated with surgery via the pterional approach except our previous research and the study of Eriksen et al. Olfactory nerves may be damaged in the frontobasal section or at medial temporal lobe levels depending on the employment of retractors. Such damage may lead to irreversible lesions. However, traumatic anosmia may recover even after 5 years. Eriksen et al. believed that lesions lasting 35 months or more must be considered as permanent.

In our previous studies, we have stressed on the importance of the preservation of olfactory nerve functions. It was possible to preserve the olfactory nerve function at a rate of 79 per cent in the cases with pituitary gland adenoma. Two articles have already been published on olfactory nerve function following operations on anterior circulation cerebral aneurysms by the front temporal approach. There are some papers on the lesions of olfactory tract brought about by the bifrontal interhemispheric approach to the ACoA aneurysms. Eriksen et al. carried out a quantitative study on Anosmia which developed as a result of the front temporal approach in a group of 25 patients who had anterior cerebral circulation aneurysms. They determined an 89 per cent Anosmia incidence on the side where surgical operation was carried out. Pool reported bilateral transection of olfactory nerves by bilateral frontal craniotomy and the interhemispheric approach. Suzuki et al. proved that it could be possible to have a 65 per cent bilateral or unilateral protection of the olfactory tract in $110 \mathrm{ACoA}$ aneurysms which they operated on by the bifrontal approach. However, Suzuki et al. achieved preservation of olfactory sense in $80 \%$ of their patients by dissecting the olfactory tract from the frontal lobe.

We observed olfactory dysfunction (absent or reduced) in 15 cases out of $100(15 \%)$. In other words, it was possible for us to preserve the functions of olfactory nerve at a rate of 85 per cent. In the operations performed, we placed the brain retractor only in the frontal sections and the retractional aperture was only 1 to 1.5 centimeters. We conclude that keeping this aperture within these limits is the most significant factor affecting postoperative olfactory nerve functions. ${ }^{2}$

In the pterional approaches, postoperative Anosmia appears to be an important risk. Brain retractor is often placed across the olfactory nerve causing strain on the nerve as well as the olfactory striae leading to the frontal and temporal lobes. The olfactory nerve may be pulled out of the cribriform plate as a result of excessive frontal lobe retraction. Also, because of the ischemic effect, even mild retractory pressure on cranial nerves may lead to temporary or permanent lesions. It was possible for us to preserve the olfactory nerve function at a rate of 79 per cent in the cases with pituitary gland adenoma. In the operations performed, we placed the retractor only in the frontal lobe and the retractional aperture was only $1-1 .^{5}$ centimeters. Keeping this retractional aperture within these limits and the intermittent retraction technique are the most significant factors affecting the postoperative olfactory nerve function in the pterional approach. ${ }^{1-2}$ For more superiorly sited ACoAs and associated aneurysms, it may be necessary to dissect the olfactory nerve from the olfactory sulcus. ${ }^{1}$

\section{Heubner artery}

It is important that the lamina terminalis cistern be adequately opened. Because, perforating arteries and the recurrent artery of Heubner need to be well identified prior to clipping the anterior communicating artery aneurysm. Especially, in the ACoAAneurysms, the aim of the neurosurgeon is to dissect and identify both $\mathrm{A} 1$ segments, both Heubner's arteries, both A2 segments, both fronto-orbital and 
both fronto-polar arteries, and the hypothalamic branches, before clipping the aneurysm. The possibility of anomalous configurations of the ACA and ACoA complex must be considered. ${ }^{1-7}$ Furthermore, the multiple variations and anomalies of the circle of Willis which are found more frequently in patients with cerebral aneurysm must be recognized and considered if vital arteries are not to be injured during operation. It should be considered of Heubner Artery Variations, originating points, different features and coursing patterns. During the closure of craniotomy, the two anterior burr holes which may produce some cosmetic defect may be filled with bone shavings to provide the normal contour ${ }^{1}$.

\section{Acknowledgments}

None.

\section{Conflicts of interest}

The authors declare there are no conflicts of interest related to the article.

\section{References}

1. Aydin IH, Takci E, Kadioglu HH, et al. Pitfalls in the pterional approach to parasellar area (review). Minim Invas Neurosurg. 1995;38(4):146-153.

2. Aydin IH, Onder A, Kadioglu HH, et al. Postoperative anosmia after Removal of pituitary adenomas using the pterional approach. Acta Neurochir (Wien). 1992;119(1-4):101-103.
3. Aydin IH, Takci E, Kadioglu HH, et al. The Variations of lenticulostriatal arteries inmiddle cerebral artery aneurysms. Acta Neurochir (Wien). 1996;138(5):555-559.

4. Aydin IH, Kadioglu HH, Tuzun Y, et al. Postoperative anosmia after anterior communicating artery aneurysms surgery by using the pterional approach. Minim Invasive Neurosurg. 1996;39(3):71-73.

5. Aydin IH, Kadioglu HH, Tuzun Y, et al. The variations of sylvian veins and cisterns in anterior circulation aneurysms. Acta Neurochirur (Wien). 1996;138(12):1380-1385.

6. Aydin IH, Takci E, Kadioglu HH, et al. Vascular variations associated with anterior communicating artery aneurysms-an Intraoperative study. Minim Invasive Neurosurg. 1997;40(1):17-21.

7. Aydin IH, Tuzun Y, Takci E, et al. The anatomical variations of sylvian veins and cisterns. Minim Invas Neurosurg. 1997;40(2):68-73.

8. Chehrazi BB. A temporal trans Sylvian approach to anterior circulation aneurysms. Neurosurgery. 1992;30(6):957-961.

9. Heros RC, Lee SH. The combined pterional/anterior temporal approach for aneurysms of the upper basilar complex: technical report. Neurosurgery. 1993;33(2):244-251.

10. Yasargil MG. Interfacial pterional craniotomy. In: Microneurosurgery (Vol 1). New York: Thime-Stratton; 1984. pp. 215-233.

11. Drake CG. Bleeding aneurysms of the basilar artery. Direct surgical management in four cases. J Neurosurg. 1961;18:230-238. 